\title{
Effects of Shenqi Fuzheng injection on Fas/FasL protein expression levels in the cardiomyocytes of a mouse model of viral myocarditis
}

\author{
TIANMIN WU, JINSHUI CHEN, LIUFANG FAN, WENYAN XIE, CHANGSHENG XU and HUAJUN WANG \\ Department of Traditional Chinese Medicine, The First Affiliated Hospital of Fujian Medical University, \\ Fuzhou, Fujian 350004, P.R. China
}

Received November 17, 2014; Accepted January 6, 2016

DOI: $10.3892 / e t m .2016 .3165$

\begin{abstract}
The aim of the present study was to examine the effects of Shenqi Fuzheng injection (SFI) on Fas and FasL protein expression levels in the cardiomyocytes of mice with viral myocarditis (VMC) and to explore the underlying anti-apoptotic mechanisms. A total of 120 male BALB/c mice were randomly divided into five groups as follows: Blank control group, model group, ribavirin group, low-dose SFI group and high-dose SFI group. The VMC model was established by the injection of coxsackievirus group B type 3 and saline, ribavirin or SFI was administered $30 \mathrm{~min}$ later. Cardiac samples were harvested from mice in each group on days 3, 10 and 30. Apoptosis of cardiac cells was examined using terminal deoxynucleotidyl transferase dUTP nick-end labeling, and Fas and FasL protein expression levels were detected using immunohistochemistry. Myocardial apoptosis and Fas/FasL protein expression levels were significantly increased in the model group, as compared with the blank group $(\mathrm{P}<0.01)$, whereas the apoptotic index $(\mathrm{AI})$ and Fas/FasL protein expression levels of cardiac cells in the high-dose SFI group were significantly decreased compared with those in the model group on day 10 (acute phase; $\mathrm{P}<0.01$ ). The AI and Fas/FasL protein expression levels of cardiac cells in the lowand high-dose SFI groups were also significantly decreased on day 30 (chronic phase; $\mathrm{P}<0.01$ ); however, no differences between the high- and low-dose groups were detected. In conclusion, SFI relieves VMC via the downregulation of Fas and FasL protein expression and the inhibition of cell apoptosis.
\end{abstract}

Correspondence to: Dr Jinshui Chen, Department of Traditional Chinese Medicine, The First Affiliated Hospital of Fujian Medical University, 20 Chazhong Road, Fuzhou, Fujian 350004, P.R. China E-mail: jinshui1@21cn.com

Key words: cell apoptosis, Fas/FasL protein, Shenqi Fuzheng injection, viral myocarditis, Chinese medicine

\section{Introduction}

Myocardial apoptosis is associated with a number of physiological and pathophysiological processes, and is the cytological basis of the development and progression of a number of cardiovascular diseases, including acute and chronic loss of cardiomyocytes in myocardial infarction, ischemic heart disease, reperfusion injury and various forms of cardiomyopathy; it is regulated by the expression of various genes (1-3). There are $>27$ viruses that are known to cause viral myocarditis (VMC), including coxsackie virus, parvovirus B19, enterovirus, adenovirus, rubella, polio, human immunodeficiency virus (HIV)-1, cytomegalovirus, and hepatitis A and C (4,5). Fas and FasL, which are members of the tumor necrosis factor/nerve growth factor family, have been demonstrated to have pivotal roles in the apoptosis of normal and cancerous cells (6-8). In animal models of myocardial infarction and myocardial overload, Fas levels have been demonstrated to increase 131 - and 21 -fold, respectively $(9,10)$. Furthermore, Fas mRNA levels were observed to be elevated 2-fold in newborn animal models of myocardial hypoxia (11). Fas, which is also known as Apo-1 or CD95, is a cell-surface receptor that has a key role in apoptotic signaling in various cell types (12). Fas ligand (FasL), which is also known as CD95L, is a member of the tumor necrosis factor super family that is capable of triggering an apoptotic cascade by cross-linking with its Fas receptor (8). The roles of Fas/FasL in the regulatory mechanisms and the apoptosis of myocardiocytes have not yet been elucidated.

Shenqi Fuzheng injection (SFI) is an injectable traditional Chinese herbal formulation comprising two Chinese herbs, Radix Codonopsis (dangshen), which is the root of Codonopsis pilosulae and Radix Astragali, which is the root of Astragali mongolici, that, in China, are commonly used to improve the immune functions of patients with chronic diseases in an integrative and holistic manner (13). SFI was approved by the State Food and Drug Administration of the People's Republic of China in 1999 as an antitumor injection to be manufactured and marketed in China $(14,15)$. Codonopsis pilosula and Astragalus membranaceus have previously been demonstrated to promote the apoptosis of cancer cells $(16,17)$. In addition, a review of animal studies has reported that, to a certain extent, Radix Astragali reduces pathological damage to normal mice 
sperm cell and thymus cells in mice, and inhibits their excessive apoptosis (18). Furthermore, C. pilosula has been shown to prevent and control the apoptosis of alveolar epithelium and pulmonary vascular endothelial cells in burned mice via the Fas/FasL pathway (19). Aqueous extracts of A. membranaceus increase immunity by upregulating Bc1-2 expression and inhibiting cell apoptosis (20). Furthermore, these aqueous extracts are capable of inhibiting necrosis and the apoptosis of neurons following ischemia/reperfusion injury; therefore, they may provide protective effects for the treatment of acute phase stroke (20).

The present study established mouse models of myocardial apoptosis by the injection of coxsackievirus group B type 3 (CVB3). CVB3 damages myocardial cells directly, or indirectly through autoimmune reactions, leading to the degeneration and necrosis of myocardial cells or interstitial inflammatory cell infiltration and fibrosis (21). Fas and FasL are apoptosis-inducing proteins that play an important role in the clearance of $\mathrm{T}$ cells, the regulation of lymphatic activation and in autoimmune processes via cytotoxic T lymphocytes (22). The therapeutic effects of SFI were investigated in the mouse models of VMC by assessing myocardial apoptosis and the expression levels of Fas and FasL.

\section{Materials and methods}

Preparation of virus and animal models. Nancy strain CVB3 was purchased from the Wuhan Institute of Virology of the Chinese Academy of Sciences (Wuhan, China). A total of 120 male healthy BALB/c mice, aged 5 weeks and weighing 16-20 g, were provided by Shanghai SLAC Experimental Animals Co., Ltd. [Shanghai, China; animal quality license, SCXK (HU) 2007-0005]. Mice were randomly divided into five groups as follows: Blank control group $(n=20)$, negative control group $(n=28)$, ribavirin group $(n=24)$, low-dose SFI group $(\mathrm{n}=24)$ and high-dose SFI group $(\mathrm{n}=24)$. Blank control group was administered $0.1 \mathrm{ml}$ Dulbecco's modified Eagle medium (Gibco; Thermo Fisher Scientific, Inc., Waltham, MA, USA) without CVB3 and after $0.5 \mathrm{~h}, 0.4 \mathrm{ml} \mathrm{NaCl}(0.9 \%)$ was injected into the abdominal cavity and mice were provided with a normal diet for 30 days. The remaining four groups were injected with $5 \times 10^{2} 50 \%$ tissue culture infective dose of CVB3 fluid $(0.1 \mathrm{ml})$ and, after $0.5 \mathrm{~h}, 0.4 \mathrm{ml} \mathrm{NaCl}(0.9 \%)$, $0.4 \mathrm{ml}$ ribavirin, $0.4 \mathrm{ml} \mathrm{SFI}$ and $0.9 \mathrm{ml} \mathrm{SFI}$, respectively, and were provided with normal diet for 30 days.

Reagents. Terminal deoxynucleotidyl transferase dUTP nick-end labeling (TUNEL) assay kits were purchased from Roche Diagnostics (Basel, Switzerland). Rabbit anti-Fas (sc-1024) and FasL (sc-834) primary polyclonal antibody and biotinylated FasL secondary antibody (sc-834) kits were purchased from Santa Cruz Biotechnology, Inc. (Dallas, TX, USA). Antibody dilution-protection fluid was purchased from Applygen Technologies, Inc., (Beijing, China), and 3,3'-diaminobenzidine (DAB) kits were purchased from Beijing Zhongshan Golden Bridge Biotechnology Co., Ltd. (Beijing, China).

Experimental drugs. SFI containing Radix Codonopsis, astragalus, and the formulation additives sodium chloride and sodium metabisulfite was produced by Lizhu Group
Limin Pharmaceutical Factory (Shaoguan, China), and each $250 \mathrm{ml}$ SFI contained $10 \mathrm{~g}$ Radix Codonopsis and Astragalus, respectively. Ribavirin (Virazole ${ }^{\circledR}$ ) was purchased from Tianjin Kingyork Group Co., Ltd., (Tianjin, China) and supplemented with normal saline to form a $0.6825 \mathrm{mg} / \mathrm{ml}$ ribavirin solution prior to abdominal injection.

Sampling of experimental animals. The protocol of the study was approved by the Animal Ethics Committee and University Committee on the Use and Care of Animals of Fujian Medical University (Fuzhou, China), in accordance with the guidelines outlined by the Ethics Committee of the Ministry of Health of China. Cardiac tissue samples were harvested from randomly chosen mice, following sacrifice via spinal dislocation at 8:00 a.m. on days 3, 10 and 30. Samples were fixed using $10 \%$ paraformaldehyde and sliced to $4-6 \mu \mathrm{m}$ following paraffin embedding to be used for TUNEL and immunohistochemical detection.

Detection of cardiac cell apoptosis using TUNEL. Following conventional dewaxing and hydration, cardiac muscle sections were incubated with proteinase $\mathrm{K}(20 \mathrm{mg} / \mathrm{ml}$ in Tris/ $\mathrm{HCl}$, $\mathrm{pH}$ 7.4-8.0) for $15 \mathrm{~min}$ at room temperature. TUNEL reaction mixture (50 $\mu \mathrm{lTdT}$ and $450 \mu \mathrm{l}$ fluorescein-dUTP) was added, the sections were placed in a humidified box at $37^{\circ} \mathrm{C}$ for $60 \mathrm{~min}$ and the sections were analyzed using immunofluorescence under an Olympus BH2 fluorescence microscope (Olympus Corporation, Tokyo, Japan). Transforming agent was added, and the sections were incubated in humidified box at $37^{\circ} \mathrm{C}$ for $30 \mathrm{~min}$. Sections were developed by incubation with $\mathrm{DAB}$ at room temperature, which was monitored using an Olympus BH2 fluorescence microscope. Subsequently, the sections were counterstained using hematoxylin (Abcam, Cambridge, MA, USA), washed with tap water, dehydrated, treated with xylene and sealed with neutral rubber. Apoptotic cells were stained brown and visualized under an Olympus BH2 fluorescence microscope. From each section, the mean of five fields of view was selected and the apoptotic index (AI) was calculated using Motic Images Advanced computed imaging software (version 3.2; Motic, Xiamen, China) as follows: AI $(\%)=($ number of positive nuclei/total number of nuclei) x 100 .

Detection of Fas and FasL protein expression levels using immunohistochemistry. Following dewaxing and hydration using gradient alcohol, sections were incubated for $10 \mathrm{~min}$ with 3\% hydrogen peroxide at room temperature in order to inhibit endogenous peroxidase activity. Fas and FasL were subjected to heat-induced antigen retrieval in Tris-EDTA (pH 9.0) buffer and citrate buffer, respectively. Sections were incubated with anti-Fas $(\mathrm{X}-2 \mathrm{O} ; 1: 25)$ and anti-FasL $(\mathrm{N}-2 \mathrm{O}$; 1:40) primary antibodies overnight at $4^{\circ} \mathrm{C}$ in a humidified box. Following washing with five times with PBS for $2 \mathrm{~min}$, the sections were incubated with biotinylated FasL secondary antibody (no dilution) for $20 \mathrm{~min}$ at room temperature. Horseradish peroxidase-conjugated avidin-biotin was subsequently added and the sections were incubated for $20 \mathrm{~min}$ at room temperature. Mean integral optical density of positive expression was measured in five randomly selected visual fields using Image Pro Plus 6.0 software (Media Cybernetics, Inc., Rockville, MD, USA). 


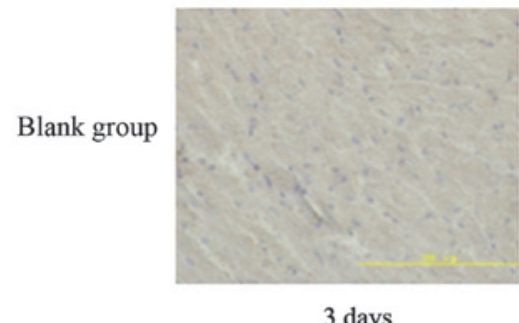

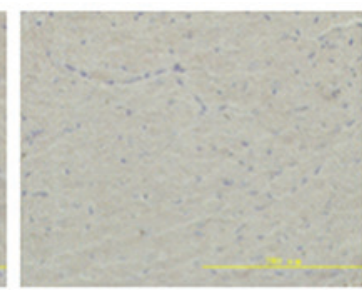

10 days

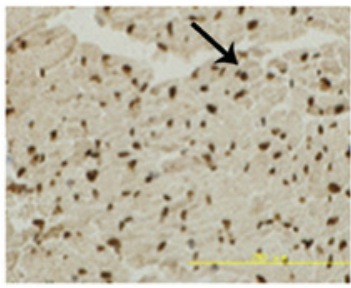

3 days

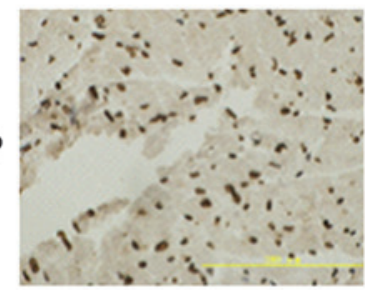

3 days

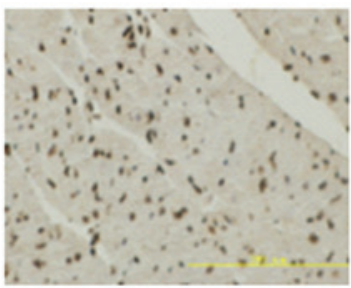

3 days

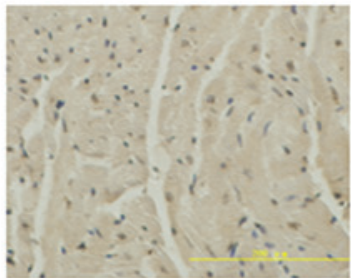

3 days

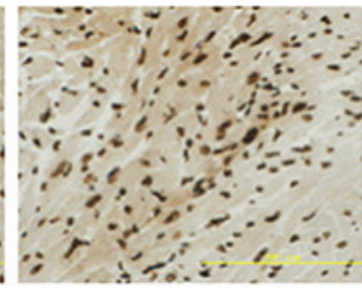

10 days

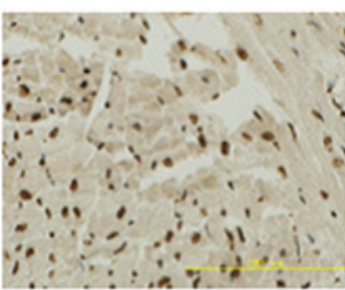

10 days

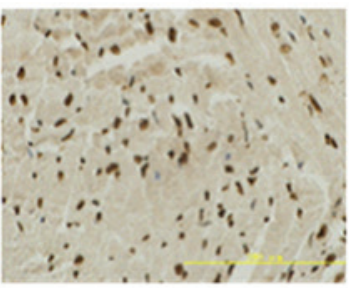

10 days

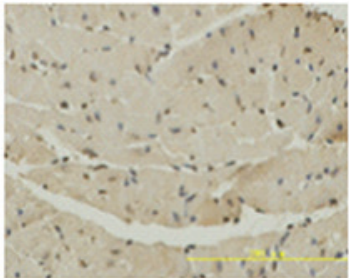

10 days

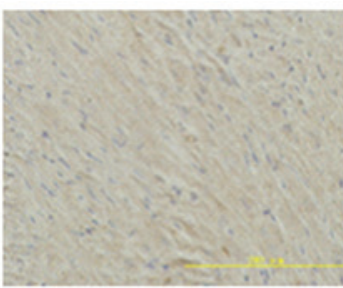

30 days

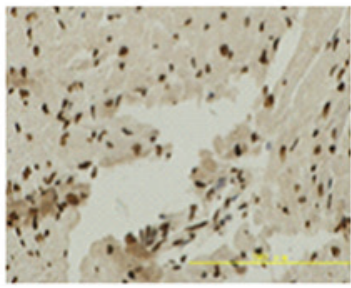

30 days

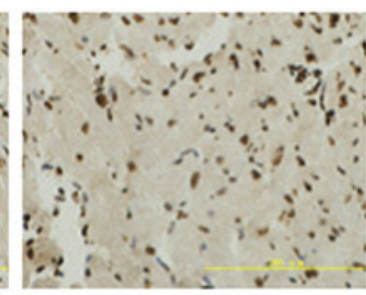

30 days

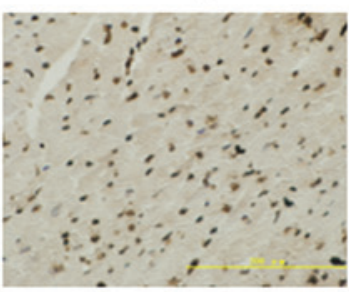

30 days

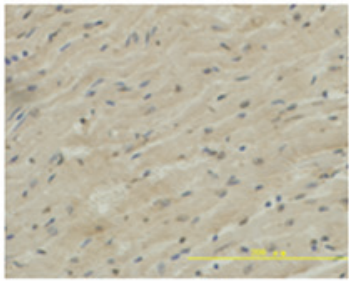

30 days

Figure 1. Representative images of the apoptotic cardiac muscle cells in each group at various time points, as detected by terminal deoxynucleotidyl transferase (TdT) dUTP nick-end labeling (magnification, x400). Positive immunohistological staining of apoptotic cells is indicated by an arrow.

Statistical analysis. Statistical analysis was performed using SPSS software, version 13.0 (SPSS, Inc., Chicago, IL, USA). All the data are expressed as the mean \pm standard deviation. Intergroup differences were compared using one way-analysis of variance, whereas between group comparisons were performed using least significant difference test. $\mathrm{P}<0.05$ was considered to indicate a statistically significant difference.

\section{Results}

Myocardial apoptosis in the mice at various time points. Myocardial apoptosis was identified by the presence of brown apoptotic cells, rounded nuclear karyopyknosis and oval brown masses (Fig. 1). In the blank control group, normal myocardial cells were detected; the nuclei were pale blue and nuclear pyknosis was not observed. However, in the model group, a large number of cardiac muscle cells contained brown round or oval nuclei and meniscus-shaped chromatin, which are characteristic of apoptosis. Strong TUNEL positive staining was detected in the nucleus, with minimal staining detected in the cytoplasm. The brown masses and changed to the nuclei became more evident at day 10, but cells appeared less apoptotic by day 30 . In the virally infected cells, lowand high-dose SFI administration induced a reduction in the number of apoptotic cells in comparison with the model group at various time points, with the high-dose SFI group exhibiting the most evident difference from the model group (Fig. 1). 
Effects of SFI on the apoptosis of myocardial cells in the mouse model of VMC. In the model group, the AI of cardiomyocytes was significantly increased, as compared with the blank group at all three time points $(\mathrm{P}<0.01)$. The AI of myocardial cells in each therapy group was not significantly reduced at day 3 (acute phase), as compared with the model group; however, the AI was significantly reduced by varying degrees on day 10 (acute phase; ribavirin and high-dose SFI, $\mathrm{P}<0.01$; low-dose SFI, $\mathrm{P}<0.05$ ). The greatest reduction was detected in the high-dose SFI group, which showed a significant difference compared with the ribavirin group $(\mathrm{P}<0.05)$. However, no significant difference was detected between the ribavirin and low-dose SFI groups on day 10. A significant reduction in the AI of cardiomyocytes was detected at day 30 in the high- and low-dose SFI groups as compared with the model group $(\mathrm{P}<0.01)$; however, no significant differences were detected between these groups. The low- and high-dose SFI groups exhibited significant differences from the ribavirin group on day 30 ( $\mathrm{P}<0.05$; Fig. 2 and Table I).

Immunohistochemical analysis of Fas/FasL proteins in the myocardial cells of the mice at various time points. Fas protein expression was demonstrated by homogeneous brown staining of the cell membrane and cytoplasm and a lack of nuclear staining (Fig. 3, as indicated by an arrow). FasL protein expression was demonstrated by homogeneous brown staining or dark brown particles located in the cell membrane and cytoplasm, and a lack of nuclear staining (Fig. 4, as indicated by an arrow). Low expression levels of Fas and FasL proteins were detected in the blank group at each time point. The greatest amount of brown staining was detected in the model group on day 3 , and no significant reduction in the amount was detected in the therapy groups. A significant increase in the amount of brown staining was detected in each group, with the exception of the blank group, on day 10, with the greatest increase demonstrated in the model group, and the smallest increase in the high-dose SFI group. The low-dose SFI and ribavirin groups exhibited more evident deposits of brown particles. At day 30 , the number of brown particles was reduced in all the groups, with the greatest reductions detected in the high- and low-dose SFI groups.

Effects of SFI on the expression levels of Fas and FasL proteins in the myocardial cells of a mouse model of VMC. A significant increase in the expression levels of Fas and FasL proteins was detected in the model group, as compared with the blank group at all time points $(\mathrm{P}<0.01)$. No significant reduction in Fas and FasL protein expression levels was detected in each of the therapy groups, as compared with the model group on day 3 (acute phase); however, all the groups demonstrated a reduction in the expression levels of Fas and FasL on day 10 (acute phase), to varying degrees ( $\mathrm{P}<0.01$ or $\mathrm{P}<0.05)$. The high-dose SFI group demonstrated the largest reductions compared with the model group $(\mathrm{P}<0.01)$, and also exhibited significant differences in Fas $(\mathrm{P}<0.01)$ and FasL $(\mathrm{P}<0.05)$ as compared with the ribavirin group $(\mathrm{P}<0.01)$. No significant differences were detected between the low-dose SFI and ribavirin groups. At day 30 (chronic phase), both the low- and high-dose SFI groups demonstrated significant reductions in the expression levels of Fas and FasL compared with the model group, although no
Table I. Effects of Shenqi Fuzheng injection (SFI) on the apoptotic index in a mouse model of viral myocarditis.

\begin{tabular}{lccl}
\hline Group & Day 3 & Day 10 & Day 30 \\
\hline Blank control & $0.32 \pm 0.09$ & $0.27 \pm 0.13$ & $0.29 \pm 0.18$ \\
Model & $1.54 \pm 0.17^{\mathrm{a}}$ & $6.83 \pm 0.16^{\mathrm{a}}$ & $4.40 \pm 0.17^{\mathrm{a}}$ \\
Ribavirin & $1.09 \pm 0.28^{\mathrm{b}}$ & $4.84 \pm 0.30^{\mathrm{a}, \mathrm{c}}$ & $3.80 \pm 0.24^{\mathrm{a}}$ \\
Low-dose SFI & $1.21 \pm 0.22^{\mathrm{b}}$ & $5.49 \pm 0.11^{\mathrm{a}, \mathrm{d}}$ & $2.40 \pm 0.33^{\mathrm{a}, \mathrm{c}, \mathrm{f}}$ \\
High-dose SFI & $0.91 \pm 0.16^{\mathrm{b}}$ & $3.43 \pm 0.17^{\mathrm{a}, \mathrm{c}, \mathrm{e}}$ & $2.00 \pm 0.25^{\mathrm{a}, \mathrm{c}, \mathrm{f}}$ \\
\hline
\end{tabular}

Data are presented as the mean \pm standard deviation. ${ }^{\mathrm{a}} \mathrm{P}<0.01$ and ${ }^{\mathrm{b}} \mathrm{P}<0.05$, vs. the blank control group; ${ }^{\mathrm{C}} \mathrm{P}<0.01$ and ${ }^{\mathrm{d}} \mathrm{P}<0.05$, vs. the model group; ${ }^{\mathrm{e}} \mathrm{P}<0.01$ and ${ }^{\mathrm{f}} \mathrm{P}<0.05$, vs. the ribavirin group.

significant differences were detected between the two SFI groups. Furthermore, Fas and FasL protein expression levels were significantly lower in the low- and high-dose SFI groups, as compared with the ribavirin group $(\mathrm{P}<0.05$ and $\mathrm{P}<0.01$, respectively) (Figs. 5 and 6; Tables II and III, respectively).

\section{Discussion}

SFI contains two Chinese medicinal herbs: Radix Astragali, which is commonly used as an immunomodulating agent in the treatment of immunodeficiency diseases and to alleviate the adverse effects of chemotherapeutic drugs, and Radix Codonopsis, which is typically used to treat dyspepsia, fatigue, bronchitis, cough, inflammation (23).

Myocarditis is considered to belong to the VMC category of traditional Chinese medicine (TCM) (19). VMC is most common among individuals with deficiencies of both energy and Yin Qi who are vulnerable to 'fire due to yin-deficiency' (FFYD), also known as 'Re Shang Qi' or 'Re Shang Yi Yin'; therefore, these are contributing factors for the development of VMC (17). A study conducted by Cao and Yin (24) demonstrated that combined energy and Yin Qi deficiency is the main mechanism of VMC pathogenesis, which affects the whole disease progress; therefore, insufficiency of heart-qi and yang (IHQY) therapy is the predominant basic treatment option for VMC. Since Qi (vital energy) can be generated in body fluid, it may be the basis and driving force for the production and transportation of fluid. Furthermore, Qi may implement fluid functions and, therefore, variability in the activity of Qi may stimulate the excretion or re-production of fluid (25). Since Qi helps to transport fluid between cells, it regulates the excretion of fluid and nourishes the lungs, thus preventing the over-excretion of fluids. The TCM, Qi and fluids maintain the balance of water metabolism, thus making SFI the preferred treatment option (25). As a TCM, Chen Qi infusion is a formulation that predominantly consists of Codonopsis pilosula and Astragalus. C. pilosula contains polysaccharides, multiple amino acids and various other inorganic elements; whereas the Astragalus genus contains saponins, polysaccharides, flavonoids, flavonoid analogs and multiple amino acids (24). In TCM, Radix Codonopsis and Astragali are compatible, with beneficial effects on the spleen and kidney, protecting and strengthening the functions of the body. Current understanding 


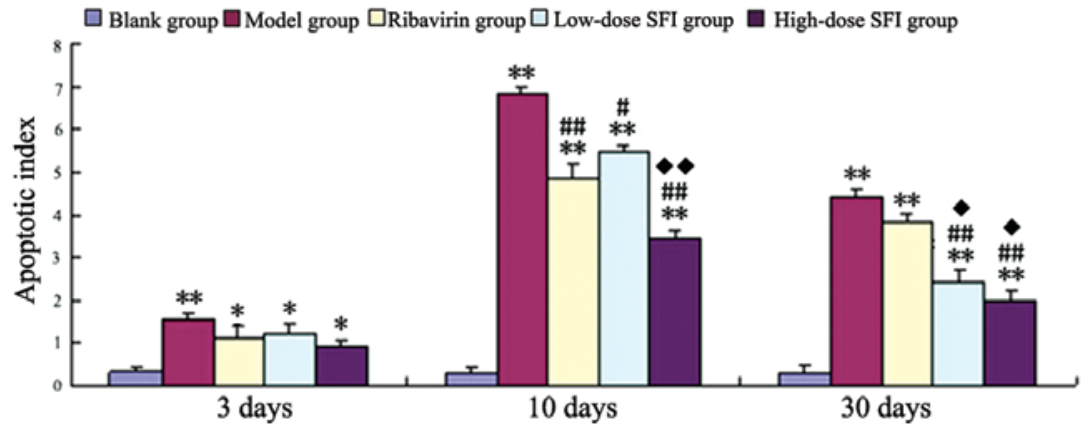

Figure 2. Effects of Shenqi Fuzheng injection (SFI) on the apoptotic index of cardiac muscle cells in a mouse model of viral myocarditis. ${ }^{*} \mathrm{P}<0.05$ and ${ }^{* *} \mathrm{P}<0.01$ vs. the blank control group; ${ }^{\#} \mathrm{P}<0.05$ and ${ }^{\# \#} \mathrm{P}<0.01$ vs. the model group; ${ }^{\oplus} \mathrm{P}<0.05$ and ${ }^{*} \mathrm{P}<0.01$ vs. the ribavirin group.

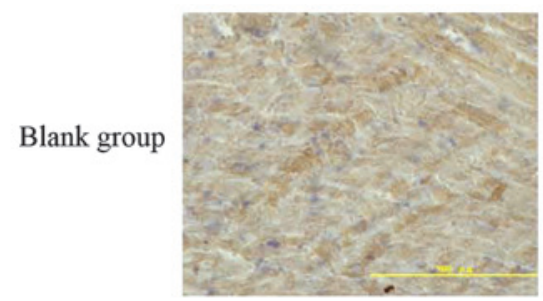

3 days

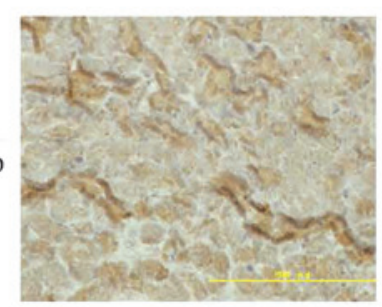

3 days

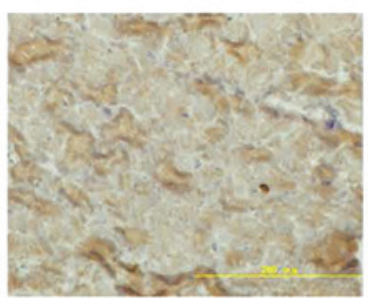

3 days

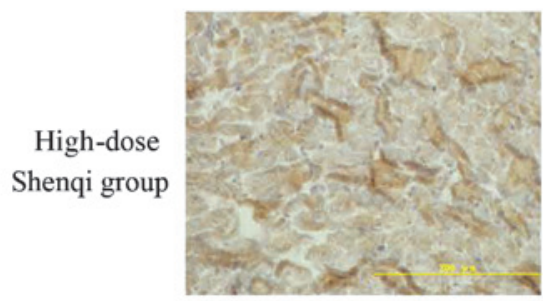

3 days

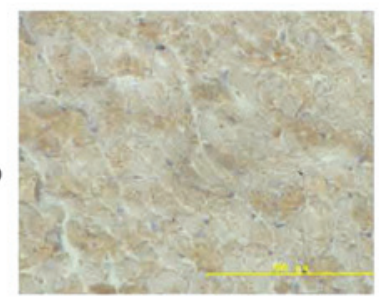

3 days

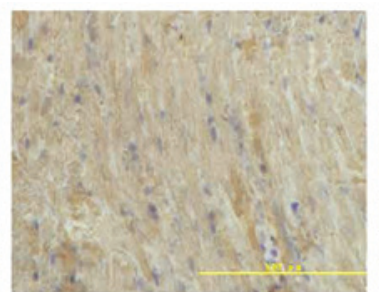

10 days

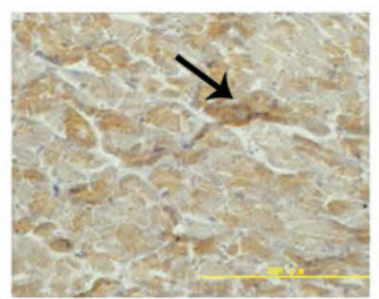

10 days

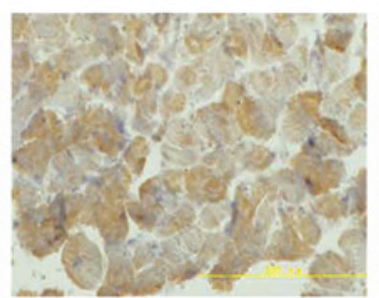

10 days

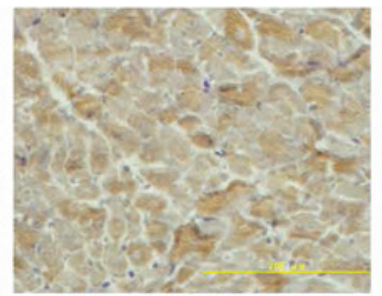

10 days

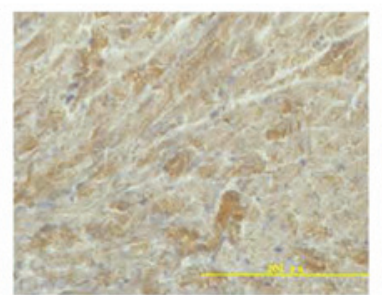

10 days

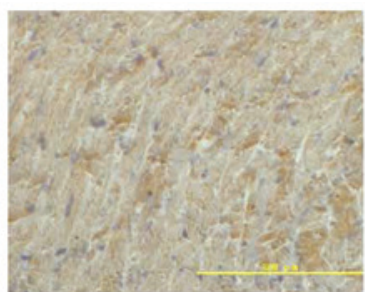

30 days

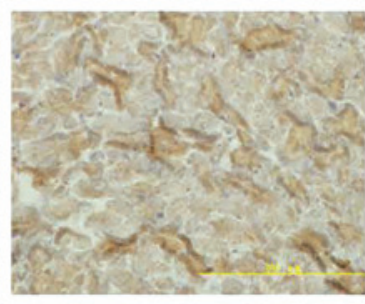

30 days

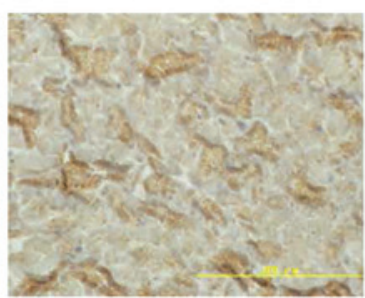

30 days

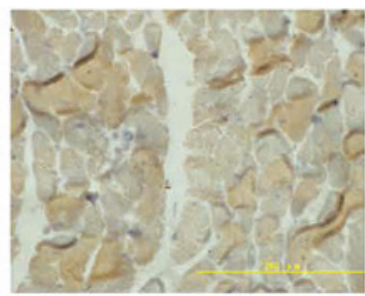

30 days

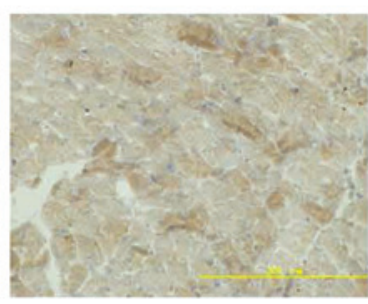

30 days

Figure 3. Representative images of the expression levels of Fas proteins at various time points in each group, as detected by immunohistochemical staining (magnification, x400). Positive immunohistological staining of Fas protein is indicated by an arrow. 


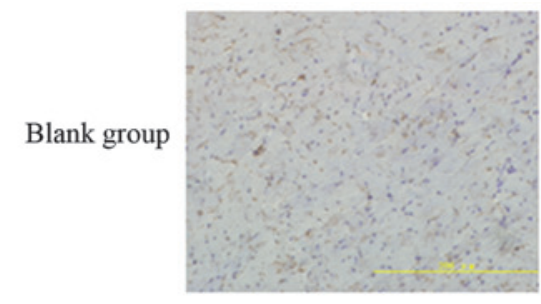

3 days

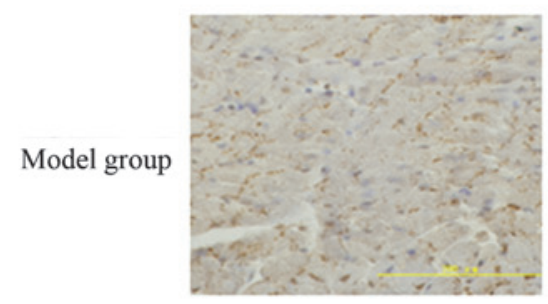

3 days

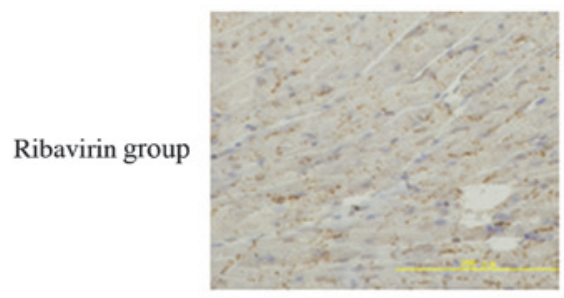

3 days

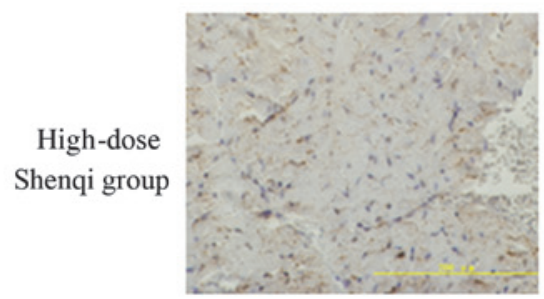

3 days

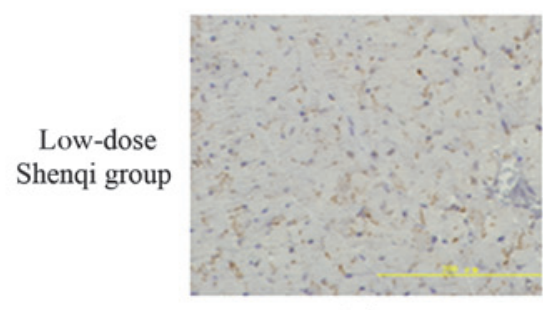

3 days

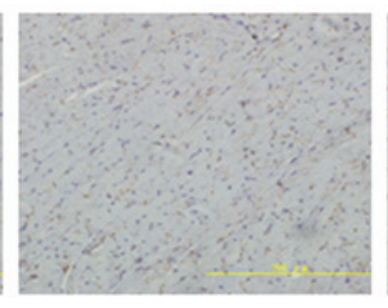

10 days

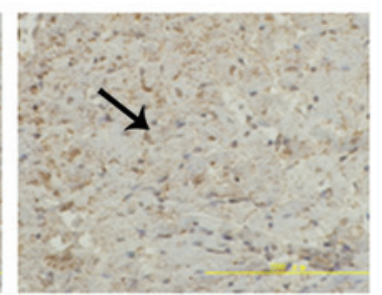

10 days

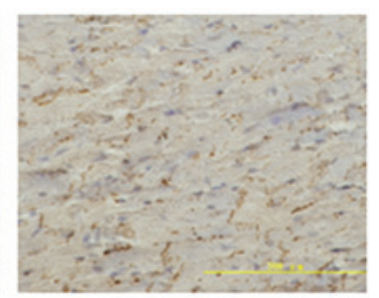

10 days

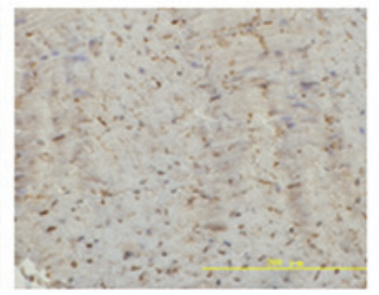

10 days

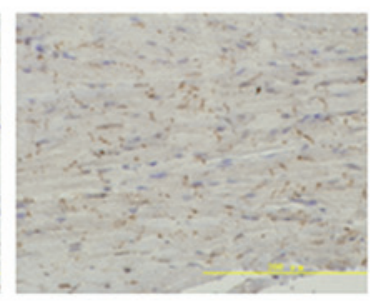

10 days

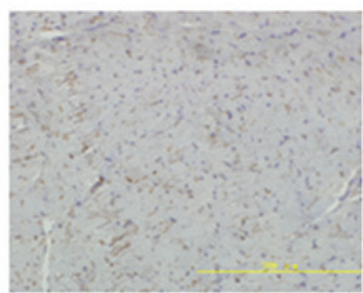

30 days

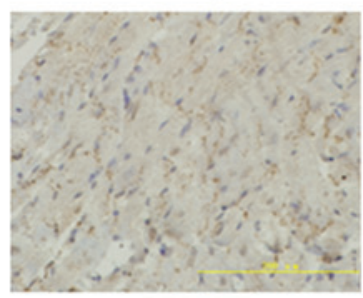

30 days

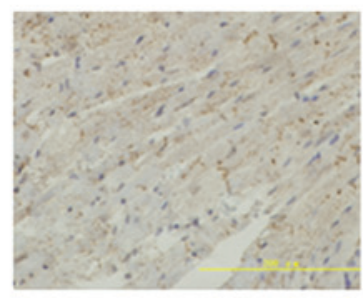

30 days

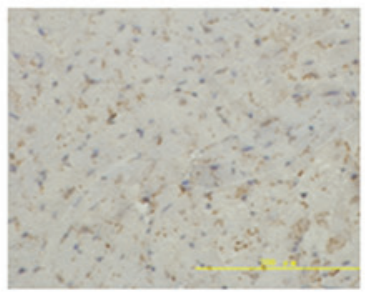

30 days

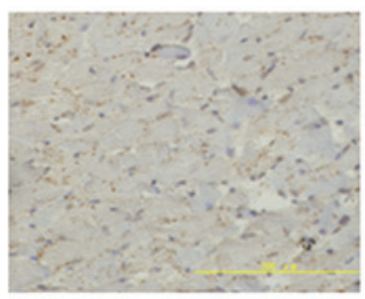

30 days

Figure 4. Representative images of the expression levels of FasL proteins at various time points in each group, as detected by immunohistochemical staining (magnification, x400). Positive immunohistological staining of FasL protein is indicated by an arrow.

of the pathogenesis of VMC remains poor; however, animal and in vivo experiments, established using coxsackie B group viruses, have indicated that damage following the infection and the immune response generated are the main mechanisms of VMC pathophysiology (26). The apoptotic changes of cells during VMC have garnered considerable attention worldwide in recent years. The Fas/FasL system is of particular interest, as it is a direct initiator of the signaling pathway during apoptosis and has an important role in the apoptosis of cardiac muscle cells (27).

The results of the present study demonstrated that the AI and expression levels of Fas and FasL proteins were increased in the model group, as compared with the blank control group, indicating that apoptosis induced by the Fas/FasL system is involved in the development and progression of VMC. In the acute phase, AI and Fas/FasL protein expression levels were reduced in the high-dose SFI group, as compared with the other groups, suggesting that SFI may inhibit the expression of Fas/FasL proteins in a dose-dependent manner, thus inhibiting apoptosis. During the chronic phase, the low-dose SFI group demonstrated decreased AI and decreased expression levels of Fas/FasL proteins compared with the model group; however, a dose-dependent trend was not detected. Considering that the protective roles of SFI to 'Zheng Qi' (the ability of the body 
Table II. Effects of Shenqi Fuzheng injection (SFI) on expression levels of Fas protein in a mouse model of viral myocarditis.

\begin{tabular}{lccc}
\hline Group & Day 3 & Day 10 & Day 30 \\
\hline Blank control & $1,817.54 \pm 450.86$ & $1,644.11 \pm 648.06$ & $1,697.95 \pm 588.73$ \\
Model & $9,685.17 \pm 793.86^{\mathrm{a}}$ & $21,984.39 \pm 2,474.56^{\mathrm{a}}$ & $16,572.54 \pm 2,463.92^{\mathrm{a}}$ \\
Ribavirin & $5,082.85 \pm 588.02^{\mathrm{b}}$ & $11,670.44 \pm 1,203.63^{\mathrm{a}, \mathrm{c}}$ & $11,949.95 \pm 2,778.89^{\mathrm{a}}$ \\
Low-dose SFI & $6,369.82 \pm 437.53^{\mathrm{a}}$ & $13,275.05 \pm 2,736.71^{\mathrm{a}, \mathrm{d}}$ & $7,782.85 \pm 438.33^{\mathrm{a}, \mathrm{c}, \mathrm{e}}$ \\
High-dose SFI & $4,793.96 \pm 629.65^{\mathrm{b}}$ & $5,782.85 \pm 692.68^{\mathrm{a}, \mathrm{c}, \mathrm{f}}$ & $5,329.74 \pm 502.10^{\mathrm{a}, \mathrm{c}, \mathrm{f}}$ \\
\hline
\end{tabular}

Data are presented as the mean \pm standard deviation. ${ }^{a} \mathrm{P}<0.05$ and ${ }^{\mathrm{b}} \mathrm{P}<0.01$ vs. the blank control group; ${ }^{\mathrm{c}} \mathrm{P}<0.05$ and ${ }^{\mathrm{d}} \mathrm{P}<0.01$ vs. the model group; ${ }^{\mathrm{e}} \mathrm{P}<0.05$ and ${ }^{\mathrm{e}} \mathrm{P}<0.01$ vs. the ribavirin group.

Table III. Effects of Shenqi Fuzheng injection (SFI) on expression levels of FasL protein in a mouse model of viral myocarditis.

\begin{tabular}{lccc}
\hline Group & Day 3 & Day 10 & Day 30 \\
\hline Blank control & $2,775.22 \pm 815.23$ & $3,622.22 \pm 723.71$ & $3,091.64 \pm 906.83$ \\
Model & $10,121.14 \pm 1,142.29^{\mathrm{a}}$ & $18,643.45 \pm 1,261.34^{\mathrm{a}}$ & $14,001.09 \pm 1,273.42^{\mathrm{a}}$ \\
Ribavirin & $7,114.32 \pm 603.02^{\mathrm{b}}$ & $13,210.27 \pm 815.73^{\mathrm{a}, \mathrm{c}}$ & $12,023.18 \pm 660.12^{\mathrm{a}}$ \\
Low-dose SFI & $8,131.18 \pm 722.24^{\mathrm{b}}$ & $14,802.36 \pm 964.35^{\mathrm{a}, \mathrm{c}, \mathrm{d}}$ & $9,433.29 \pm 551.72^{\mathrm{a}, \mathrm{c}, \mathrm{e}}$ \\
High-dose SFI & $7,921.25 \pm 512.43^{\mathrm{b}}$ & $10,536.04 \pm 1,480.64^{\mathrm{a}, \mathrm{c}, \mathrm{e}}$ & $8,419.39 \pm 507.09^{\mathrm{a}, \mathrm{c}, \mathrm{f}}$ \\
\hline
\end{tabular}

Data are presented as the mean \pm standard deviation. ${ }^{a} \mathrm{P}<0.01$ and ${ }^{\mathrm{b}} \mathrm{P}<0.05$ vs. the blank control group; ${ }^{\mathrm{c}} \mathrm{P}<0.01$ and ${ }^{\mathrm{d}} \mathrm{P}<0.05$ vs. the model group; ${ }^{\mathrm{e}} \mathrm{P}<0.05$ and ${ }^{\mathrm{f}} \mathrm{P}<0.01$ vs. the ribavirin group.

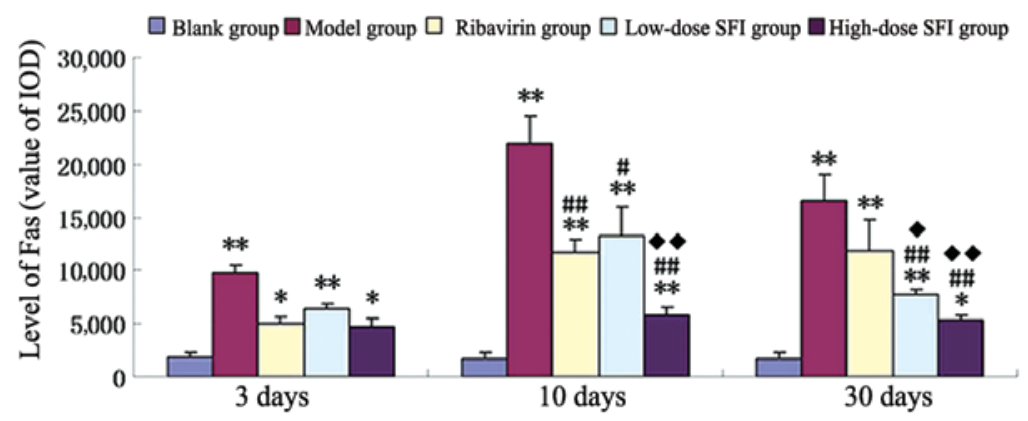

Figure 5. Effects of Shenqi fuzheng injection (SFI) on the expression levels of Fas protein in the various murine groups. ${ }^{*} \mathrm{P}<0.05$ and ${ }^{* *} \mathrm{P}<0.01$ vs. the blank control group; ${ }^{\#} \mathrm{P}<0.05$ and ${ }^{\# \#} \mathrm{P}<0.01$ vs. the model group; ${ }^{*} \mathrm{P}<0.05$ and ${ }^{~}{ }^{*} \mathrm{P}<0.01$ vs. the ribavirin group. IOD, integrated optical density.

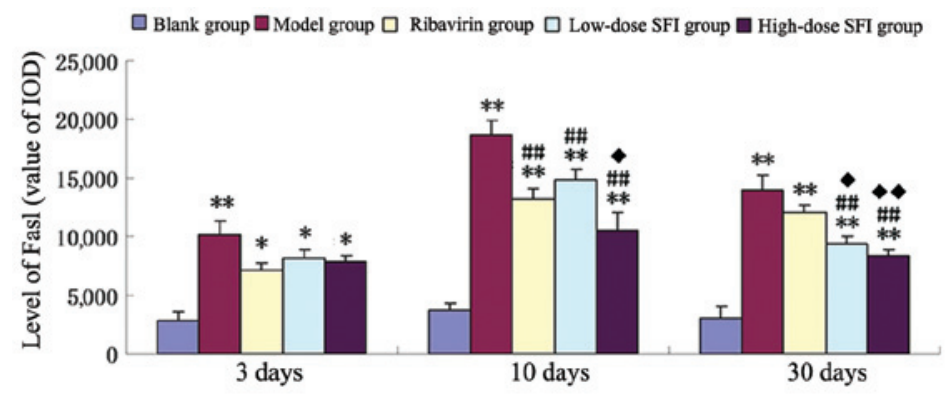

Figure 6. Effects of Shenqi fuzheng injection (SFI) on the expression levels of FasL protein in the various murine groups. ${ }^{*} \mathrm{P}<0.05$ and ${ }^{* *} \mathrm{P}<0.01$ vs. the blank control group; ${ }^{\# \#} \mathrm{P}<0.01$ vs. the model group; ${ }^{\circ} \mathrm{P}<0.05$ and ${ }^{"} \mathrm{P}<0.01$ vs. the ribavirin group. IOD, integrated optical density.

to resist disease) are involved, potentially meaning appropriate dose is sufficient to protect Zheng Qi. It has previously been demonstrated that A. membranaceus plus supportive therapy is effective at improving myocardial enzymes, abnormal electrocardiogram and cardiac function, and SFI is effective at improving the immune system of mice $(23,28)$. There are 
currently three basic therapeutic approaches for the treatment of VMC using TCM, including excretory function enhancement, cardiovascular system improvement and tonic effect reinforcement.

The present study had certain limitations and further robust trials are required in order to explore the use of herbal medicines in the treatment of VMC. In particular, the specific immunological and cytotoxic mechanisms require investigation, and the main effective components of the formulation should be identified. Furthermore, any potential interactions between the components require investigation.

In conclusion, apoptosis induced by the Fas/FasL system is associated with the pathogenesis of VMC, and may be pivotal. SFI inhibited the apoptosis of cardiac cells and may have a therapeutic role in the treatment of VMC by downregulating the expression of Fas/FasL proteins. Therefore, the results of the present study suggested that SFI may be used to treat VMC; however, the mechanisms underlying the Fas/FasL-downregulating effects of SFI and the subsequent apoptosis of cardiac cells require further elucidation.

\section{Acknowledgements}

The authors would like to thanks the Professor Fund at The Education Department of Fujian Province (grant no. JS07013) for support.

\section{References}

1. Kang PM and Izumo S: Apoptosis in heart: Basic mechanisms and implications in cardiovascular diseases. Trends Mol Med 9: 177-182, 2003.

2. Gottlieb RA, Burleson KO, Kloner RA, Babior BM and Engler RL: Reperfusion injury induces apoptosis in rabbit cardiomyocytes. J Clin Invest 94: 1621-1628, 1994.

3. Olivetti G, Abbi R, Quaini F, Kajstura J, Cheng W, Nitahara JA, Quaini E, Di Loreto C, Beltrami CA, Krajewski S, et al: Apoptosis in the failing human heart. N Engl J Med 336: 1131-1141, 1997.

4. Kearney MT, Cotton JM, Richardson PJ and Shah AM: Viral myocarditis and dilated cardiomyopathy: Mechanisms, manifestations, and management. Postgrad Med J 77: 4-10, 2001

5. Cooper LT Jr: Myocarditis. N Engl J Med 360: 1526-1538, 2009.

6. Suda T, Takahashi T, Golstein P and Nagata S: Molecular cloning and expression of the Fas ligand, a novel member of the tumor necrosis factor family. Cell 75: 1169-1178, 1993.

7. Ashkenazi A and Dixit VM: Death receptors: Signalling and modulation. Science 281: 1305-1308, 1998.

8. Krammer PH: The CD95 (APO-1/Fas) CD95L system. Toxicol. Lett 103: 131-137, 1998.

9. Cheng W, Li B, Kajstura J, et al: Stretch-induced programmed myocyte cell death. J Clin Invest 96: 2247-2259, 1995.
10. Kajstura J, Cheng W, Reiss K, Clark WA, Sonnenblick EH, Krajewski S, Reed JC, Olivetti G and Anversa P: Apoptotic and necrotic myocyte cell deaths are independent contributing variables of infarct size in rats. Lab Invest 74: 86-107, 1996.

11. Yaniv G, Shilkrut M, Lotan R, Berke G, Larisch S and Binah O: Hypoxia predisposes neonatal rat ventricular myocytes to apoptosis induced by activation of the Fas (CD95/Apo-1) receptor: Fas activation and apoptosis in hypoxic myocytes. Cardiovasc Res 54: 611-623, 2002.

12. Monroe EW: Desloratidine for the treatment of chronic urticaria. Skin Therapy Lett 7: 1-5, 2002.

13. Lu Y and Lu YX: Clinical application and pharmacology function of Shenqi fuzheng injection. Shizhen Guo Yi Guo Yao 17: 2083-2083, 2006 (In Chinese).

14. Pan L: The practice of digital traditional Chinese medicine of Shenqi Fuzheng injection. Zhongguo Chu Fang Yao 1: 37-39, 2009 (In Chinese).

15. Zhong ZH: Oral history: The industrialization of Shenqi Fuzheng injection - from proved recipe to SFDA approved new drug. Zhongguo Chu Fang Yao 1: 33-36, 2009 (In Chinese).

16. Li Y, Mai GR, Chen BF and LiT: The relationship between the apoptosis mediated by Fas/Fasl ligand system and virus myocarditis in mice. Shi Yong Er Ke Lin Chuang Za Zhi 17: 631-632, 2002 (In Chinese).

17. Wang SH, Wang Q and Liu BC: Experience on the treatment of viral myocarditis through the lungs. Xin Zhong Yi 39: 15-16, 2007.

18. Luo H, Han M and Liu JP: Systematic review and meta-analysis of randomized controlled trials of Chinese herbal medicine in the treatment of Sjogren's syndrome. Zhong Xi Yi Jie He Xue Bao 9: 257-274, 2011 (In Chinese).

19. Jiao HJ: The pharmacology efficacy and clinical application about dangshen. Chinese Journal of Clinical Medicine 25: 89-92, 2005.

20. Shao BM, Xu W, Dai H, Tu P, Li Z and Gao XM: A study on the immune receptors for polysaccharides from the roots of astragalus membranaceus, a Chinese medicinal herb. Biochem Biophys Res Commun 320: 1103-1111, 2004.

21. Zhang MX and Cao HQ: Effects of Yi qi compound on cardiac muscle cells of CVB3 viral myocarditis. Zhong Guo Zhong Yi Yao Xin Xi Za Zhi She 8: 35-38, 2011 (In Chinese).

22. Bi MR: The association between Fas expression and the prognosis of myocarditis. Shandong Da Xue Xue Bao 4: 60-61, 2006 (In Chinese).

23. Xu CR and Zhou JH: Astragalus injection in the treatment of acute viral myocarditis. Yi Xue Li Lun Yu Shi Jian 22: 655-656, 2009 (In Chinese)

24. Cao $\mathrm{HH}$ and Yin $\mathrm{HJ}$ : Clinical practice of Yi qi yang yin therapy on viral myocarditis. Zhong Yi Jiao Yu 18: 52-53, 1999.

25. Sun GY, Tong Y, Chen KW and Li QZ: Basic theory of TCM. Beijing: Zhongguo Zhong Yi Yao Za Zhi 8: 150-153, 2002.

26. Zhang JH and Zhang YL: Shenqifuzheng infusion treats viral myocarditis - observational study of 32 patients. Zhongguo Meitan Gongye Yixue Zazhi 4: 580, 2010 (In Chinese).

27. Mei H, Ren SM and Bo L: Role of Fas/Fas ligand apoptotic pathway of peripheral blood lymphocytes in the development of viral myocarditis. Zhongguo Xiao Er Ji Jiu Yi Xue 4: 320-322, 2011 (In Chinese).

28. Niu RY, Yan XY and Wang JD: Effects of Shenqi Fuzheng injection on the immune function in mice. Shanxi Nong Ye Ke Xue 36: 86-88, 2008 (In Chinese). 